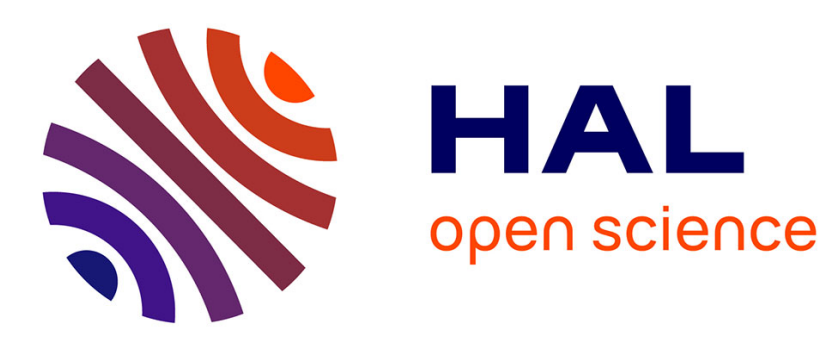

\title{
An experimental investigation and a simple model of valveless pump
}

Thomas Bringley, Stephen Childress, Nicolas Vandenberghe, Jun Zhang

\section{To cite this version:}

Thomas Bringley, Stephen Childress, Nicolas Vandenberghe, Jun Zhang. An experimental investigation and a simple model of valveless pump. Physics of Fluids, 2008, 20 (3), pp.033602. 10.1063/1.2890790 . hal-00327520

\section{HAL Id: hal-00327520 \\ https://hal.science/hal-00327520}

Submitted on 7 Sep 2015

HAL is a multi-disciplinary open access archive for the deposit and dissemination of scientific research documents, whether they are published or not. The documents may come from teaching and research institutions in France or abroad, or from public or private research centers.
L'archive ouverte pluridisciplinaire HAL, est destinée au dépôt et à la diffusion de documents scientifiques de niveau recherche, publiés ou non, émanant des établissements d'enseignement et de recherche français ou étrangers, des laboratoires publics ou privés. 


\title{
An experimental investigation and a simple model of a valveless pump
}

\author{
Thomas T. Bringley, ${ }^{1, a)}$ Stephen Childress, ${ }^{1}$ Nicolas Vandenberghe, ${ }^{1,2}$ and Jun Zhang ${ }^{3,1}$ \\ ${ }^{1}$ Applied Mathematics Laboratory, Courant Institute of Mathematical Sciences, New York University, \\ 251 Mercer Street, New York, New York 10012, USA \\ ${ }^{2}$ IRPHE, Université de Provence, 13384 Marseille, France \\ ${ }^{3}$ Department of Physics and Center for Soft Matter Research, New York University, 4 Washington Place, \\ New York, New York 10003, USA
}

(Received 20 December 2006; accepted 11 February 2008; published online 24 March 2008)

\begin{abstract}
We construct a valveless pump consisting of a section of elastic tube and a section of rigid tube connected in a closed loop and filled with water. By periodically squeezing the elastic tube at an asymmetric location, a persistent flow around the tubes is created. This effect, called the Liebau phenomenon or valveless pumping, has been known for some time but is still not completely understood. We study the flow rates for various squeezing locations, frequencies, and elastic tube rigidities. To understand valveless pumping, we formulate a simple model that can be described by ordinary differential equations. The time series of flow velocities generated by the model are qualitatively and quantitatively similar to those seen in the experiment. The model provides a physical explanation of valveless pumping, and it allows us to identify the essential pumping mechanisms. (C) 2008 American Institute of Physics. [DOI: 10.1063/1.2890790]
\end{abstract}

\section{INTRODUCTION}

Beginning in 1954, Liebau published a series of papers describing a novel kind of water pump. ${ }^{1-3}$ One version of his pump consisted of two tubes, one relatively elastic and one relatively rigid, connected in series to form a closed loop and filled with water. When the elastic tube was squeezed periodically at an asymmetric location along its length, a flow around the tubes developed. The direction of the flow depended on the location of the squeezing; the flow always traveled from the longer portion of the elastic tube toward the shorter portion.

This effect became known as the Liebau phenomenon, and the pump became known as a valveless pump. It is not immediately clear why such a pump would create a flow, nor is it clear why the flow would go in the observed direction. Traditional pumps use valves to restrict the fluid motion to one direction or, by peristalsis, push the fluid in one direction. The tube walls in a valveless pump move normal to the flow and do not select a preferred direction. Moreover, the motion of the tube walls is symmetric in time, so that if one direction of flow is preferred during closing, the opposite direction ought to be preferred during opening, so that on average, there is no obvious preferred direction.

In this paper, we describe a valveless pump similar to that used by Liebau. We use a mechanical forcing mechanism to squeeze the elastic tube at various locations and frequencies, and we also test elastic tubes of different rigidities. Our observations are generally consistent with those of Liebau. At high frequencies, a large mean flow is generated in the rigid section in the direction observed by Liebau. Generally, the flow strength increases with frequency and with distance of the forcing mechanism from the center of the elastic tube. We observe resonances at some frequencies and flow

$\overline{\left.{ }^{a}\right)}$ Electronic mail: bringley@cims.nyu.edu. direction reversals at low frequencies. A diagram showing our experimental setup can be seen in Fig. 1 in Sec. II. The arrows in this diagram indicate the flow direction that Liebau observed.

This paper also describes a simple model of valveless pumping. In the model, the pump is conceived as consisting of four discrete regions: The section of the rigid tube, the section of the elastic tube that is squeezed, and the two elastic sections on either side of the squeezed section. The states of these regions are given by scalar variables such as fluxes, volumes, and pressures. These states evolve according to coupled linear ordinary differential equations that we derive from the fluid equations and from basic assumptions. All parameters are measured experimentally or derived from physical principles. Though some of our approximations are crude, this simple model produces a flow in the loop as a function of time that is remarkably similar to that seen in the experiment for a wide range of frequencies, forcing positions, and elastic tube rigidities. A deficiency of the model is that it does not produce the resonances seen in the experiment.

The model includes several small nondimensional parameters, which allow us to perform an asymptotic analysis in a distinguished limit. This analysis reveals the leading behavior of the four regions of the pump and produces an approximate expression for the average flow in terms of the parameters of the model. We define a measure of efficiency for the pump, and we find that at large Reynolds numbers, a valveless pump can be more efficient than a traditional pump, but that it is otherwise inefficient. We also identify the essential physical mechanisms that create flow and select the preferred direction.

Our model is valid in the regime where the time required for waves to travel the length of the elastic tube is much shorter than the pumping period. In this regime, we can ef- 
fectively average over the dynamics of individual waves by considering the regions of the elastic tube as having uniform pressures that represent the effects of the waves. Our experiments fall into this regime. If the pumping frequency were much greater, the elastic tube much longer, or the wave speed, which is proportional to the square root of the tube's rigidity, much slower, our model would break down and a one-dimensional or higher model including wave dynamics would be needed.

While a higher-dimensional model could also be used for the regime of our experiment, we show that a far simpler model is sufficient to capture much of the behavior of the pump. Although relatively low dimensional, our model (1) accurately reproduces experimental results, (2) provides a closed-form expression for the flux through the pump, and (3) admits a clear physical explanation for valveless pumping in the regime tested.

Liebau was motivated by his interest in the human circulatory system. The human heart has very efficient valves which ensure that blood flows from the heart into arteries and is returned by veins. Liebau was interested in patients with malfunctioning aortic valves but who could still circulate blood. ${ }^{1-3}$ Arteries are much less compliant than veins; ${ }^{4}$ analogously, the shorter portion of the elastic tube is more resistant to changes in volume than the longer portion. If this analogy holds, the observations of Liebau suggest that the contraction of the heart, even without valves, would generate a circulation from veins to arteries, the desired direction.

There are other possible applications related to blood circulation. The thoracic pump theory of cardiopulmonary resuscitation (CPR) proposes that the valves of the heart are inoperative and the heart acts as a passive conduit during CPR. Valveless pumping could act as the mechanism that creates circulation. A summary of this theory and competing theories along with references can be found from Jung and Peskin. ${ }^{5}$ In the early stages of development, the human fetus has a functioning circulatory system, though its heart has not yet formed chambers or valves. It is commonly thought that blood is circulated by a peristaltic mechanism, though recent experiments in living zebra fish embryos show evidence that valveless pumping plays a role. ${ }^{6}$

Valveless pumping may have applications in engineering as well. Rinderknecht et al. have constructed a successful valveless micropump similar to that described above. ${ }^{7}$ Micropumps are thought to be a promising application of valveless pumping because valveless pumps are easy to construct and require few moving parts.

Since Liebau, valveless pumping has generated much interest. Further experiments confirming the observations of Liebau were performed by Kilner ${ }^{8}$ and Hickerson et al. ${ }^{9}$ The latter, using a somewhat different experimental setup, found resonances and flow reversals at high frequencies. Jung and Peskin used the immersed boundary method to create twodimensional numerical simulations of a valveless pump. ${ }^{5}$ They also observed resonances and flow reversals.

One-dimensional models of valveless pumps have been used by Thomann, ${ }^{10}$ Ottesen, ${ }^{11}$ Manopoulus et al.,${ }^{12}$ Borzì and Propst, ${ }^{13}$ and Auerbach et al. ${ }^{14}$ Hickerson and Gharib recently proposed a model that reduces the dynamics to mo-

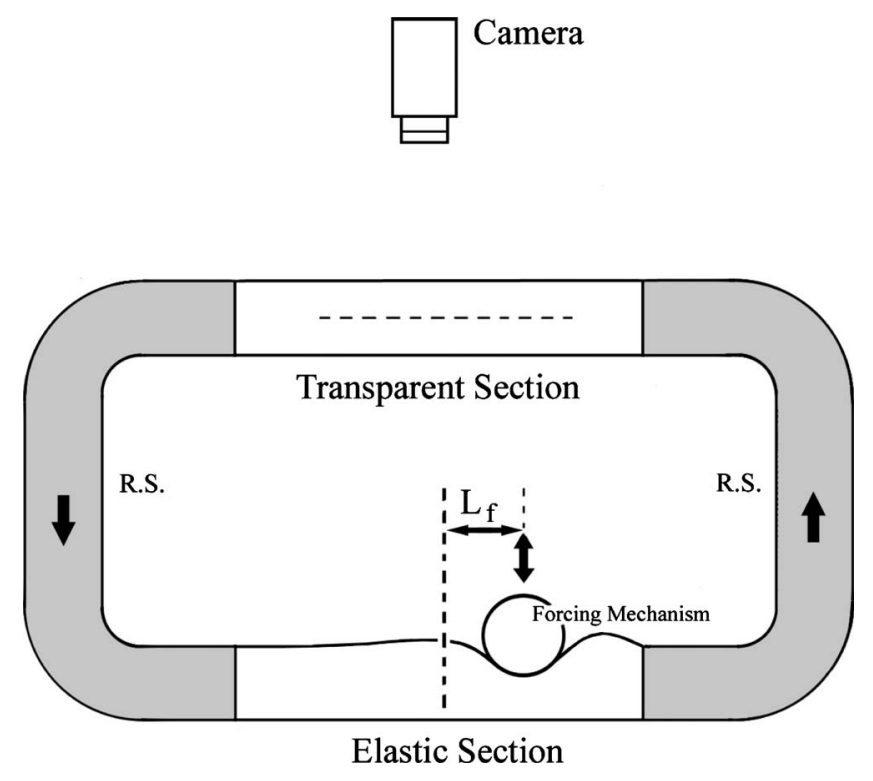

FIG. 1. Diagram of the experimental setup. An elastic tube is connected to a rigid tube as shown. R.S. indicates rigid sections. The tubes are filled with water. A cylinder depresses the elastic tube periodically. The movement of flow visualization particles in a transparent section of the rigid tubing is captured by a video camera. The illuminated portion of the transparent section is indicated by the dashed line. The arrows in the rigid sections indicate the direction of flow observed by Liebau, which we confirm to be the primary flow direction.

tion of discrete waves in the elastic tube that impart momentum to the fluid in the rigid tube such that a net flow is created. $^{15}$

A zero-dimensional model of a valveless pump similar to our model was proposed by Moser et al. ${ }^{16}$ Our model differs from theirs in that ours is derived from basic fluid equations and includes extra terms that come from the nonlinearity in these equations. Our analysis shows that these terms are essential for flow creation, meaning that valveless pumping is a truly nonlinear phenomenon. This is a fundamental difference between their results and ours, which we will not try to reconcile in this paper.

The structure of this paper is as follows. In Sec. II, we describe the experiment and present its results in Sec. III. In Sec. IV, we construct the model. The model is integrated numerically, and the results are described in Sec. V. We analyze the model in Sec. VI and, in Sec. VII, we interpret the model physically.

\section{DESCRIPTION OF THE EXPERIMENT}

The experimental apparatus is a closed, fluid-filled loop. The loop consists of two sections: A short flexible tube and a long rigid tube. It is constructed by connecting two pieces of relatively rigid polyvinyl chloride (PVC) tubing of equal length to both a section of latex tubing and a section of transparent rigid plastic tubing, as shown in Fig. 1. This loop is filled completely with water. The setup is built with lateral symmetry, so that pumping results from asymmetry in the forcing, not the apparatus. Some important physical dimensions of our setup are listed in Table I. 
TABLE I. Physical constants and parameters used throughout the paper.

\begin{tabular}{|c|c|c|}
\hline Symbol & Value & Parameter indicated \\
\hline$\rho$ & $1.0 \mathrm{gm} / \mathrm{cm}^{3}$ & Density of water \\
\hline$\mu$ & $0.01 \mathrm{gm} / \mathrm{cm} \mathrm{s}$ & Viscosity of water \\
\hline$A_{r}$ & $2.85 \mathrm{~cm}^{2}$ & Cross-sectional area of the rigid tube \\
\hline$L_{r}$ & $124.2 \mathrm{~cm}$ & Total length of the rigid tube \\
\hline$A_{0}$ & $2.85 \mathrm{~cm}^{2}$ & Rest cross-sectional area of the elastic tube \\
\hline$h_{0}$ & 0.051 and $0.081 \mathrm{~cm}$ & Wall thicknesses of the elastic tubes \\
\hline$E$ & $0.99 \mathrm{MPa}$ & Young's modulus of the elastic tubes \\
\hline$Y$ & $0.0063 \mathrm{MPa} s$ & Damping parameter of the elastic tubes \\
\hline$L_{p}$ & $2.22 \mathrm{~cm}$ & Length of the pumping region \\
\hline$L_{f}$ & Various & Forcing offset \\
\hline$L_{1}$ & $8.7 \mathrm{~cm}+L_{f}-L_{p} / 2$ & Length of elastic region 1 \\
\hline$L_{2}$ & $8.7 \mathrm{~cm}+L_{f}-L_{p} / 2$ & Length of elastic region 2 \\
\hline$f$ & Various & Forcing frequency \\
\hline
\end{tabular}

To periodically compress the tube, we use a cylinder attached to a lever arm, as shown in Fig. 2. The lever is supported by a spring and is periodically pushed down onto the elastic tube by a motor. The spring, under compression, ensures that the lever is in contact with the motor at all times. The mechanism is configured so that the cylinder, at its lowest point, closes off the elastic tube completely. At its highest elevation, it is above the elastic tube, so that for approximately half of each cycle, the cylinder is not in contact with the tube. The forcing is characterized by its frequency $f$ and the horizontal distance of the center of the forcing cylinder from the center of the latex tube, $L_{f}$, hereafter called the forcing offset.

Forcing with a nonzero offset results in time dependent flow in the rigid part of the tube. This flow is laminar and we use particle tracking to measure the flow speed in the rigid section. Small $(57 \mu \mathrm{m})$ neutrally buoyant particles are added to the fluid. We illuminate the central vertical plane of the tube (as shown by the dashed line in Fig. 1) and we record the motion of these particles. We use a custom-written particle tracking program to measure the velocity at the center of the tube $u(t)$, the instantaneous flux $Q(t)$, the time averaged central velocity over one period $\bar{u}$, and the time averaged flux $\bar{Q}$. Additional details are provided in an endnote. ${ }^{17}$

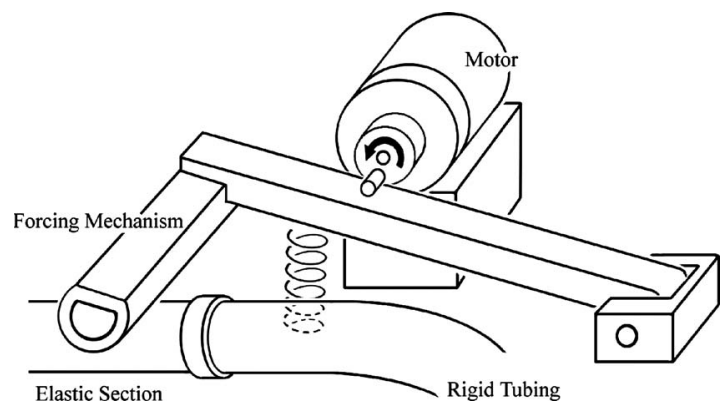

FIG. 2. Diagram of the forcing mechanism. A motor drives the periodic motion of a lever, to which a cylinder is attached, so that the cylinder compresses the elastic tube. A spring keeps the lever in contact with the motor at all times. A potentiometer at the base of the lever measures its phase within a driving period (not shown).
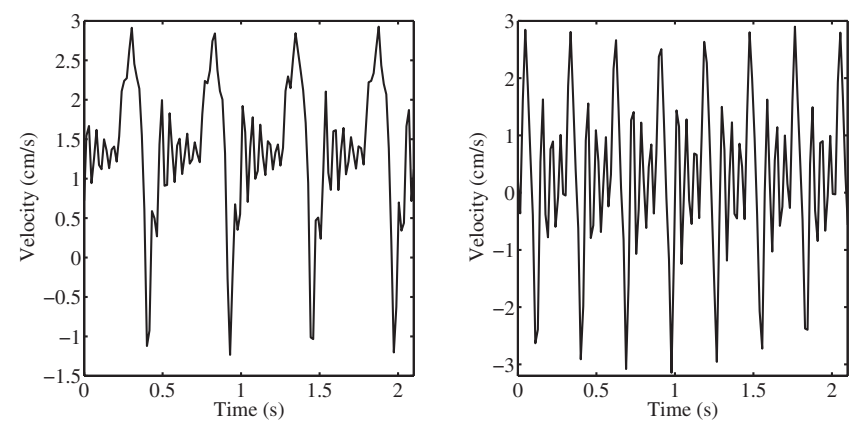

FIG. 3. Experimental results showing the velocity in the rigid section as a function of time. Left: Plot of $u$ vs time for the tube with thickness $h_{0}$ $=0.051 \mathrm{~cm}$, forcing frequency $f=1.91 \mathrm{~Hz}$, and forcing offset $L_{f}=5.66 \mathrm{~cm}$. Right: Plot of $u$ vs time for $h_{0}=0.051 \mathrm{~cm}, f=3.49 \mathrm{~Hz}$, and $L_{f}=3.27 \mathrm{~cm}$. This plot shows a resonance.

\section{RESULTS}

In an experimental run, we start with the fluid at rest and allow the pump to run until it reaches a quasisteady, periodic state, after which we perform measurements. We vary the frequency of the forcing and the forcing offset, and we use two different latex tubes with different rigidities, characterized by the tube's wall thickness $h_{0}$.

Two representative plots of $u(t)$ for different parameters can be seen in Fig. 3. Positive velocities indicate flow in the counterclockwise direction in Fig. 1, which is the direction of the flow observed by Liebau. Negative velocities indicate clockwise flow. Both plots show a high degree of periodicity in the flow.

The plot on the left is for $f=1.91 \mathrm{~Hz}$ and $L_{f}=5.66 \mathrm{~cm}$. The average velocity is positive, though $u(t)$ is sometimes negative. In each period, an acceleration is followed by a rapid deceleration and then a damped oscillation. The frequency of the damped oscillation is about $19 \mathrm{~Hz}$.

The plot on the right is for $f=3.49 \mathrm{~Hz}$ and $L_{f}=3.27 \mathrm{~cm}$. The average velocity is nearly zero, though instantaneous velocities are large in both the positive and negative directions. Again, an acceleration is followed by a rapid deceleration and then a damped oscillation, but here the period of acceleration and deceleration seems to match the frequency of the oscillation. Specifically, the time between the final peak of the damped oscillation and the peak velocity after acceleration is similar to the oscillation period. Also, the time between the most negative velocity after deceleration and the first trough of the damped oscillation is similar to the oscillation period. The period of deceleration lasts somewhat longer than half a period. We refer to this phenomenon as a resonance. We will see that, for each elastic tube rigidity, resonances occur at a particular frequency for all values of the forcing offset. These resonances greatly affect the average velocity.

Figure 4 shows how the instantaneous velocity corresponds to the phase of the forcing. The solid line shows $u(t)$ as a function of time for $f=1.91 \mathrm{~Hz}$ and $L_{f}=5.66 \mathrm{~cm}$. The dashed line shows the height of the forcing mechanism as a function of time, scaled to vary between zero and 1 and shifted so that it fits on this plot. The motion of the forcing 


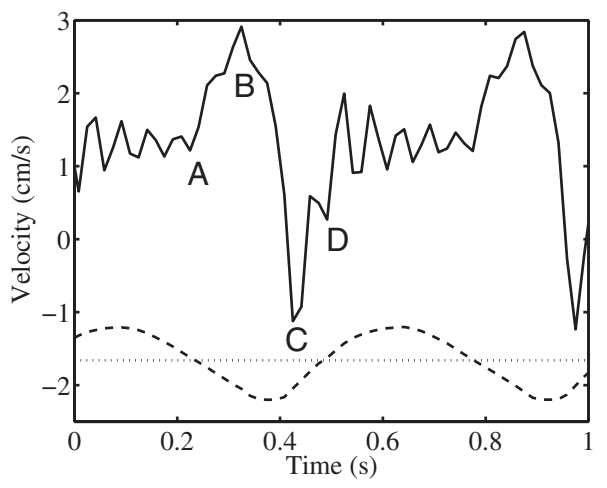

FIG. 4. Solid line: Plot of experimentally measured $u$ vs time for $h_{0}$ $=0.051 \mathrm{~cm}, f=1.91 \mathrm{~Hz}$, and $L_{f}=5.66 \mathrm{~cm}$. Dashed line: Scaled, shifted height of the forcing mechanism. Dotted line: Height at which the forcing mechanism contacts the elastic tube. At point A, the forcing mechanism contacts the elastic tube and acceleration begins. At point B, the elastic tube is nearly closed by the forcing mechanism and the flow decelerates rapidly. At point $\mathrm{C}$, the elastic tube opens and the flow accelerates again. At point $\mathrm{D}$, the forcing mechanism disengages from the elastic tube and a damped oscillation begins.

mechanism is nearly sinusoidal, and the dotted line shows the height at which it first comes into contact with the elastic tube.

Four points of interest are labeled with capital letters on the plot of $u(t)$. At point $\mathrm{A}$, the beginning of the acceleration of the flow corresponds to the time when the forcing mechanism first comes in contact with the elastic tube. The flow accelerates as the tube is compressed until point $\mathrm{B}$, when the tube is nearly closed. Then, the flow decelerates until point $\mathrm{C}$, when the tube has begun to open but is still mostly closed. The flow accelerates again as the tube opens until point D, when the forcing mechanism disengages from the elastic tube. The flow then undergoes a damped oscillation until the forcing mechanism comes down again.

Figure 5 shows $\bar{u}$ as a function of the forcing frequency for different forcing locations and different elastic tube rigidities. The plot on the left shows the full range of frequencies we test. The plot on the right zooms in on the low frequencies. Higher frequencies tend to result in greater velocities and the shapes of the velocity curves are convex. Above a frequency of about $1 \mathrm{~Hz}, \bar{u}$ is always positive, so
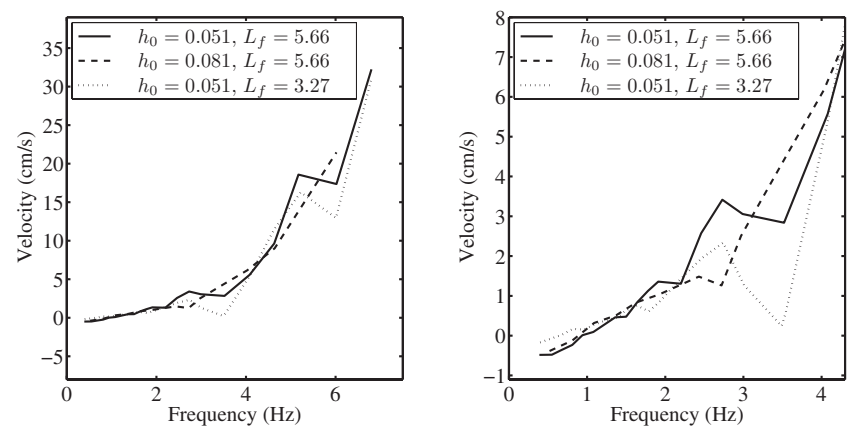

FIG. 5. Experimental results showing the average velocity in the rigid section as a function of forcing frequency. Left: Plot of $\bar{u}$ vs forcing frequency comparing results for different rigidities and forcing offsets. Right: Zoom-in of the lower frequency portion of the plot on the left.
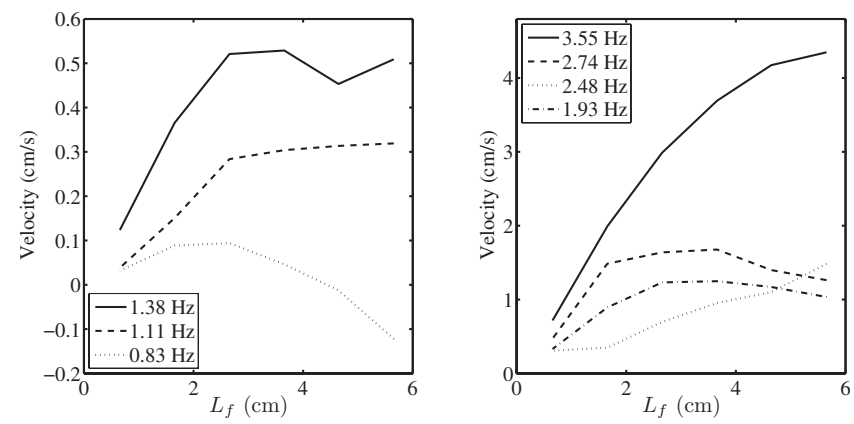

FIG. 6. Experimental results showing the average velocity in the rigid section as a function of forcing location. Left: Plot of $\bar{u}$ vs forcing offset using the tube with $h_{0}=0.081$ at various lower frequencies. Right: Same as left but at higher frequencies.

that the average flow goes in the direction observed by Liebau. For low frequencies, however, the average velocity tends to be in the opposite direction.

Using the less rigid elastic tube, we see resonances at $3.5 \mathrm{~Hz}$ for all choices of the forcing offset. The resonances decrease the magnitude of the average velocity, sometimes dramatically. We do not observe that resonances cause the average velocity to ever become negative. It may be that a sort of resonance accounts for the drop in velocity when the frequency increases from 5 to $6 \mathrm{~Hz}$, but visual evidence is inconclusive.

We observe resonances when using the more rigid tube as well. These occur at approximately $2.5 \mathrm{~Hz}$. We do not observe a drop in velocity between 5 and $6 \mathrm{~Hz}$. Other than differences in the resonances, using the more rigid elastic tube has little affect on the average velocity, decreasing the magnitude slightly at low frequencies.

Forcing closer to the center of the elastic tube, which corresponds to decreasing $L_{f}$, decreases the magnitude of the average velocity substantially at low frequencies and somewhat at high frequencies. Figure 6 shows $\bar{u}$ as a function of $L_{f}$ for the more rigid elastic tube and for fixed frequencies. At high frequencies, the velocity increases as $L_{f}$ increases. At lower frequencies, the behavior of the velocity is not monotonic. At very low frequencies, the average velocity actually changes direction as the forcing position varies. The velocity is positive for small $L_{f}$ and becomes negative for large $L_{f}$. In the plot on the right, one can see that the $2.48 \mathrm{~Hz}$ curve lies mostly below the $1.93 \mathrm{~Hz}$ curve, an effect of resonance.

We check for hysteresis in the valveless pump by raising the frequency very high, then dropping it and waiting until a periodic steady state is reached. These experiments show no evidence of hysteresis. We also check to see if our setup is symmetric by switching the forcing to the opposite side of the tube. The resulting average velocities are very nearly the opposite of the original ones, as expected. We confirm Liebau's observation that forcing in the center of the elastic tube produces no net flow. We check the stability of this result by perturbing the initial flow and then forcing in the center. Even at the highest frequencies, we find that the pump quickly settles to a state of zero net flow. 


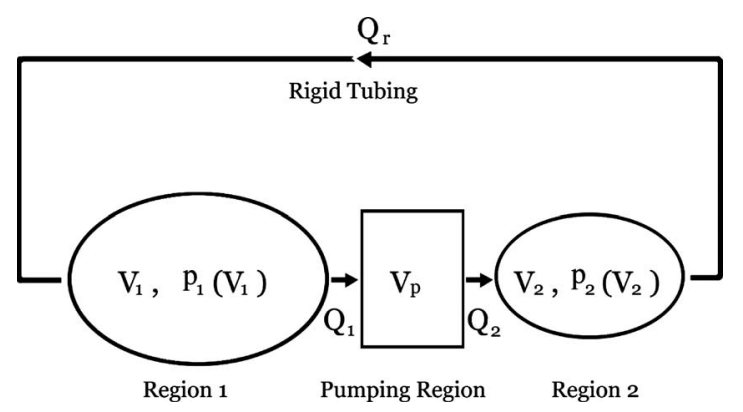

FIG. 7. Diagram showing the components and variables in our model.

\section{A SIMPLE MODEL}

We present a model of the valveless pump described above. We intend to make our model as simple as possible while still capturing the qualitative features of the experiment. Remarkably, our model is in reasonable quantitative agreement with the experiment as well. The simplicity of our model makes it accessible to analysis, and, by taking advantage of small parameters, we are able to derive an expression for the steady-state average flux in the pump. Analysis of the model also leads to a physical explanation of the mechanism that creates valveless pumping.

The model has four components: The rigid tube, the "pumping region" of the elastic tube, and the regions of the elastic tube to the left and right of the pumping region, referred to respectively, as regions 1 and 2 .

The state of the rigid tube is described by its flux $Q_{r}$. That of the pumping region is described by its volume $V_{p}$, its flux from region $1, Q_{1}$, and its flux to region $2, Q_{2}$. Our model treats the elastic regions as reservoirs that store fluid and that are at uniform pressure. We ignore wave propagation, and we do not keep track of the fluid velocity in these regions. The state of region 1 is described solely by its volume $V_{1}$ and pressure $p_{1}$; that of region 2 is described by its volume $V_{2}$ and pressure $p_{2}$. See Fig. 7 for a diagram showing the configuration of the four components. We now derive the equations of the model.

Mass conservation implies

$$
\begin{aligned}
& \dot{V}_{1}=Q_{r}-Q_{1}, \quad \dot{V}_{2}=Q_{2}-Q_{r}, \\
& \dot{V}_{p}=Q_{1}-Q_{2} .
\end{aligned}
$$

The dot signifies a time derivative. We supply the forcing to the system externally, so $V_{p}$ is taken to be a given function of time.

To derive equations for the fluxes, we use the following equations for one-dimensional, incompressible flow in a long, thin tube:

$$
\begin{aligned}
& A_{t}+(A u)_{x}=0, \\
& u_{t}+u u_{x}+\frac{p_{x}}{\rho}=\frac{\sigma}{\rho A} .
\end{aligned}
$$

The variable $x$ specifies the direction around the tube. The tube has cross-sectional area $A(x, t)$, average (over a cross section) velocity $u(x, t)$, and average pressure $p(x, t)$. The fluid density is $\rho$. The boundary stress is $\sigma(x, t)$.

These equations represent conservation of volume and momentum, respectively. They may be derived as an asymptotic limit of the full three-dimensional Navier-Stokes equations assuming that the flow is nearly unidirectional and uniform across the cross section of the tube, that $A$ is a slowly varying function of $x$, and also that the radius of the tube is small in comparison with its length and its radius of curvature. A derivation is given by Ottesen. ${ }^{11}$

These equations may be used to model elastic tubes by specifying a relationship between $p$ and $A$. We, however, treat the rigid tube and the pumping region as having specified cross-sectional areas $A(x, t)$. We must select a model for $\sigma$. To avoid introducing additional complication, we crudely approximate $\sigma$ by its value for a parabolic flow profile which is $-8 \pi \mu u$, where $\mu$ is the fluid viscosity. This approximation is crude because the Womersley number ${ }^{18}$ in our experiments, given by $\sqrt{2 f A_{r} \rho / \mu}$, is of order of 30 , so we expect the instantaneous flow profiles to differ significantly from parabolic profiles. We feel, however, that this approximation for $\sigma$ works sufficiently well for our simple model. A more realistic description, particularly in the pumping region which undergoes rapid, dramatic changes of shape, would likely involve introducing additional dynamic variables or dimensions. Even some one-dimensional models of valveless pumping use a similar prescription as ours ${ }^{11,13}$ or use an inviscid fluid. ${ }^{10,14}$

Equation (3) is a conservation law for the velocity in the tube, where the velocity flux is $u^{2} / 2+p / \rho$. In the case of no boundary stress, the integral of the velocity in the tube is conserved. If the stress acts as a drag, the average velocity will tend to decay. This represents a potential problem for one-dimensional models that use these equations everywhere if boundary conditions are not handled carefully. We shall make use of this conservation of velocity to analyze the model.

We use the tube equations to derive a dynamic equation for the flux in the rigid tube. Here, the assumptions under which the tube equations are valid are justified by our experimental observations. We assume that the pressures felt at the endpoints of the rigid tube are the pressures of regions 1 and 2. The rigid tube has length $L_{r}$ and uniform crosssectional area $A_{r}$. Because $A_{r}$ is a constant, Eq. (2) implies that the velocity is constant in $x$ and equal to $Q_{r} / A_{r}$. Equation (3) can then be integrated over the length of the tube to find an equation for $Q_{r}$,

$$
\dot{Q}_{r}=A_{r} \frac{p_{2}-p_{1}}{\rho L_{r}}-\frac{8 \pi \nu}{A_{r}} Q_{r} .
$$

The constant $\nu$ is the kinematic viscosity. The fluid in the rigid tube is accelerated by pressure differences and is simultaneously decelerated by viscous drag.

We also use the tube equations to derive an equation for the fluxes in the pumping region. The assumptions used in deriving the tube equations are probably far from valid in the pumping region, where the flow is not likely to be unidirectional and perhaps will be turbulent. Nevertheless, the equa- 
tion we derive seems to work reasonably well. A more detailed characterization of the pumping region and the boundary conditions could improve this model but may be difficult to achieve without introducing additional complications.

We treat the pumping region as if it moves in a specified manner, so that its cross-sectional area $A_{p}(x, t)$ is explicitly given. It is convenient to let $x=0$ be the center of the pumping region. We assume that $A_{p}$ is a symmetric function of $x$, so that the shape of the pumping region does not have a directional bias. We use the tube equations to find an equation for $Q_{1}$ and $Q_{2}$. Integrating Eq. (2) in the pumping region, we obtain

$$
u=\frac{Q_{p}(t)}{A_{p}}-\frac{1}{A_{p}} \int_{0}^{x} \partial_{t} A_{p}\left(x^{\prime}, t\right) d x^{\prime},
$$

where $Q_{p}(t)$ is the integration constant. Matching fluxes at the endpoints of the pumping region,

$$
\begin{aligned}
& Q_{1}=Q_{p}+\int_{-L_{p} / 2}^{0} \partial_{t} A_{p} d x^{\prime}=Q_{p}+\dot{V}_{p} / 2, \\
& Q_{2}=Q_{p}-\int_{0}^{L_{p} / 2} \partial_{t} A_{p} d x^{\prime}=Q_{p}-\dot{V}_{p} / 2,
\end{aligned}
$$

where $L_{p}$ is the length of the pumping region. We recognize $Q_{p}$ as the average flux through the pumping region,

$$
Q_{p}=\frac{Q_{1}+Q_{2}}{2} .
$$

We derive an equation for the dynamics of $Q_{p}$ by integrating Eq. (3) over the pumping region and inserting the expression for $u$ in Eq. (5). See the Appendix for details of the derivation. The resulting equation is

$$
\dot{Q}_{p}=2 \gamma(t) \frac{\dot{V}_{p}}{V_{p}} Q_{p}+\frac{V_{p}}{\alpha(t) \rho L_{p}^{2}}\left(p_{1}-p_{2}\right)-\beta(t) \frac{8 \pi \nu L_{p}}{V_{p}} Q_{p},
$$

where $\alpha, \beta$, and $\gamma$ are dimensionless geometric factors that depend only on the "shape" of the pumping region. They are

$$
\begin{aligned}
& \alpha(t)=\left\langle A_{p}\right\rangle\left\langle A_{p}^{-1}\right\rangle, \\
& \beta(t)=\alpha\left\langle A_{p}^{-2}\right\rangle\left\langle A_{p}^{-1}\right\rangle^{-2}, \\
& \gamma(t)=\frac{\left\langle A_{p}\right\rangle}{2\left\langle A_{p}^{-1}\right\rangle\left\langle\partial_{t} A_{p}\right\rangle}\left(-\left\langle\partial_{t} A_{p}^{-1}\right\rangle+\frac{\left\langle\partial_{t} A_{p}\right\rangle}{A_{p}\left(L_{p} / 2, t\right)^{2}}\right),
\end{aligned}
$$

where the brackets denote an average over $x$ in the pumping region.

By the Schwarz inequality, $\alpha \geqslant 1$, with equality when $A_{p}$ is a constant function of $x$. This is the case when the pumping region is a cylinder. Then, $\beta=1$ and $\gamma=1$ also. The degree to which $\alpha, \beta$, and $\gamma$ differ from 1 , in a way, indicates the degree to which the pumping region is not cylindrical. By the convexity of $x^{2}, \beta \geqslant \alpha$, with equality, again, when the pumping region is cylindrical. If $\partial_{t} A_{p}$ is of one sign, then $\gamma$ $>0$. When $A_{p}$ approaches zero somewhere in the pumping
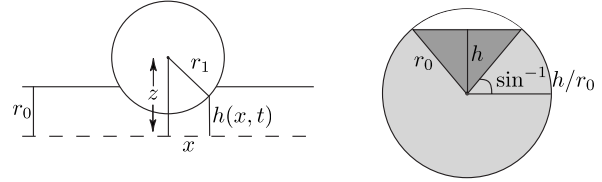

FIG. 8. Left: The elastic tube, represented by the horizontal lines, is impacted by the forcing mechanism, represented by the circle. At position $x, h$ is the vertical distance from the center of the elastic tube to the forcing mechanism. Right: Cross section of the elastic tube at position $x$. The unshaded area is that which intersects the forcing mechanism, and we let $A_{p}(x, t)$ be equal to the total shaded area. The first term in Eq. (9) corresponds to the area of the lightly shaded region, and the second term corresponds to the area of the darkly shaded region.

region, as it does in our experiment, $\alpha, \beta$, and $\gamma$ may diverge. We discuss the consequences of this divergence later in this paper.

In light of Eq. (6), Eq. (7) relates the variables in our model. The average flux $Q_{p}$ is accelerated by pressure differences across the tube and is simultaneously retarded by drag. The second term, $2 \gamma\left(\dot{V}_{p} / V_{p}\right) Q_{p}$, is unusual. It has two pieces, corresponding to the two terms in Eq. (8) for $\gamma$. The first comes from the increase or decrease of flux in the pumping region that results from an increase or decrease in its crosssectional area at fixed fluid velocity at the endpoints. The second comes from the nonlinear term in the momentum equation. To see how, notice that in the simplifying case when the pumping region is a cylinder, the nonlinear term $\left\langle A_{p} u u_{x}\right\rangle=-\left(\partial_{t} A_{p}\right)\langle u\rangle=-\left(\dot{V}_{p} / V_{p}\right) Q_{p}$. The effect of these two pieces is that $Q_{p}$ will tend to accelerate when the tube is opening and that $Q_{p}$ will tend to decelerate when the tube is closing. As we shall see, leaving out the second piece that comes from the nonlinear term in the momentum equation results in the pump having an average flow of precisely zero.

We specify an $A_{p}$ in the pumping region that mimics that imposed experimentally. In the experiment, the elastic tube, at rest, is a cylinder of radius $r_{0}$. The forcing mechanism is also a cylinder of radius $L_{p} / 2=: r_{1}>r_{0}$ oriented orthogonally to the elastic tube. Let $z(t)$ be the shortest distance between the centerlines of the two cylinders. Then, we let $A_{p}(x, t)$ be the cross-sectional area of the section of the elastic tube that does not intersect the forcing mechanism. The result is

$A_{p}(x, t)= \begin{cases}r_{0}^{2}\left(\pi / 2+\sin ^{-1} h / r_{0}\right)+h \sqrt{r_{0}^{2}-h^{2}}, & -r_{0} \leqslant h \leqslant r_{0} \\ \pi r_{0}^{2}, & r_{0}<h,\end{cases}$

where $h(x, t)=z-\sqrt{r_{1}^{2}-x^{2}}$. A short derivation of this result is shown in Fig. 8 and explained in its caption.

We let $z$ vary sinusoidally between its minimum value $r_{1}-r_{0}$, which is when the forcing mechanism closes the pump completely, and its experimentally measured maximum value, which is $r_{1}+2.7 r_{0}$.

Finally, in a thin walled cylindrical elastic tube of a Hookean elastic material, the circumferential stress in the elastic material is equal to the pressure in the fluid multiplied by the tube radius and divided by the wall thickness. According to Hooke's law, this circumferential stress is proportional 
to the change of diameter of the tube. Linearizing about the rest area $A_{0}$, we get that the pressure $p=k\left(A-A_{0}\right)$ with $k$ $=E h_{0} /\left(2 r_{0} A_{0}\right)$. In the present case, we add a viscoelastic correction to account for losses in the elastic material and we use the relation

$$
p=k\left(A-A_{0}\right)+n A_{t}
$$

with $n=Y h_{0} /\left(2 r_{0} A_{0}\right)$, where $Y$ is an intrinsic property of the viscoelastic material and is measured experimentally in a separate miniexperiment. Thus, we obtain equations for the two elastic regions,

$$
\begin{aligned}
& p_{1}=k\left(\frac{V_{1}}{L_{1}}-A_{0}\right)+n \frac{\dot{V}_{1}}{L_{1}}, \\
& p_{2}=k\left(\frac{V_{2}}{L_{2}}-A_{0}\right)+n \frac{\dot{V}_{2}}{L_{2}} .
\end{aligned}
$$

Many have focused on one-dimensional wave phenomena in these regions to explain valveless pumping. By treating the elastic regions as reservoirs, we do not capture the effects of individual waves and instead assume a uniform pressure. We claim that this approximation is justified in the regime tested in our experiment. The linearized wave speed in the elastic tube is $\sqrt{k A_{0} / \rho}$, which is more than $5 \mathrm{~m} / \mathrm{s}$ even for the less rigid elastic tube. Thus, the time required for a wave to travel the length of the elastic tube, which is only $17.4 \mathrm{~cm}$, is much less than the pumping periods we test in the experiment. Our simple treatment of the elastic regions is an adiabatic approximation which averages over the contributions of the individual fast-moving waves. This approximation will break down if the pumping frequency is much higher than those we test, if the elastic tube is much longer, or if the elastic tube is much more flexible. The frequency required for the pumping period to match the time for a wave to travel the length of the elastic tube is approximately $30 \mathrm{~Hz}$, much higher than we are able to test in the experiment.

A problem with our model is that the time scale for the oscillation of fluid between regions 1 and 2 via the pumping region is on the order of the wave time scale. As a result of ignoring delay times associated with finite wave speed, the frequency of this oscillation is too high. This effect causes the oscillation to be incorrectly overdamped. We believe that this problem also accounts for the failure of our model to reproduce the resonances. We do not, at present, know a way to correct this problem in the context of our model. A onedimensional or higher model including wave dynamics is likely needed to be fully accurate. Nevertheless, our model reproduces most of the phenomena seen in the experiment.

\section{MODEL RESULTS}

We now have a complete set of equations, Eqs. (1), (4), (6), (7), (10), and (11), and that $V_{p}$ is given. We can combine them into a system of three differential equations for the variables $Q_{r}, Q_{p}$, and

$$
V_{d}=\frac{1}{2}\left(V_{1}-L_{1} A_{0}-V_{2}+L_{2} A_{0}\right) .
$$

Physically, $V_{d}$ is the difference in the excess volumes of elastic regions 1 and 2 . The sum of these excess volumes is known because of mass conservation.

The evolution of this quantity is

$$
\dot{V}_{d}=Q_{r}-Q_{p}
$$

Using conservation of mass,

$$
\begin{aligned}
& V_{1}-L_{1} A_{0}=\frac{1}{2}\left(V_{p, 0}-V_{p}\right)+V_{d}, \\
& V_{2}-L_{2} A_{0}=\frac{1}{2}\left(V_{p, 0}-V_{p}\right)-V_{d},
\end{aligned}
$$

where $V_{p, 0}$ is the rest volume of the pumping region. Using Eqs. (10) and (11),

$$
\begin{aligned}
p_{1}-p_{2}= & \left(\frac{1}{L_{1}}+\frac{1}{L_{2}}\right)\left(k V_{d}+n \dot{V}_{d}\right) \\
& +\frac{1}{2}\left(\frac{1}{L_{1}}-\frac{1}{L_{2}}\right)\left[k\left(V_{p, 0}-V_{p}\right)-n \dot{V}_{p}\right] .
\end{aligned}
$$

Equations (4), (7), and (12), along with relation (13), make up our system of differential equations. These are linear and inhomogeneous with nonconstant, periodic coefficients.

We integrate the expression for $A_{p}$, given in Eq. (9), using the trapezoidal rule to calculate $V_{p}(t)$. We analytically differentiate Eq. (9) in time and integrate using the trapezoidal rule to find $\dot{V}_{p}$. The factors $\alpha, \beta$, and $\gamma$ are calculated similarly. When $z$ approaches its minimum value, $r_{1}-r_{0}, \alpha$, $\beta$, and $\gamma$ diverge, creating problems for our ordinary differential equation integrator. We actually let $z$ vary sinusoidally between its maximum value and $r_{1}-r_{0}+\delta$ where $\delta>0$. We found that the results converge as $\delta$ approaches zero, and, in practice, we use $\delta=0.0005 \mathrm{~cm}$. To improve efficiency, we precalculate and tabulate values of $V_{p}, \dot{V}_{p}, \alpha, \beta$, and $\gamma$ for many values of $t$ in a single pumping period. Their values at arbitrary $t$ are found using periodicity and linear interpolation.

Starting from rest, we integrate the system of differential equations using MATLAB's ODE45. For the values of the physical constants used, see Table I. We integrate until the system reaches a periodic steady state; then we calculate the average flux $\bar{Q}$ by taking an average of $Q_{r}$ over an integral number of pumping periods.

Figure 9 shows fluxes in the rigid tube, $Q_{r}$, as functions of time for various frequencies, forcing offsets, and elastic tube rigidities. Results from the model are shown in black. Those from the experiment are shown in gray. The phases of the two results have been synchronized, so that the forcing mechanism is at its minimum height at coinciding times for both model and experiment. We emphasize that no parameters of our model were chosen to fit the experimental data.

The upper left plot shows results obtained using the more rigid elastic tube, $f=0.81 \mathrm{~Hz}$, and $L_{f}=5.66 \mathrm{~cm}$. The model and experiment agree very well. As in the experiment, $Q_{r}$ accelerates when the pumping region starts to close. As the forcing mechanism reaches its minimum point, $Q_{r}$ decel- 

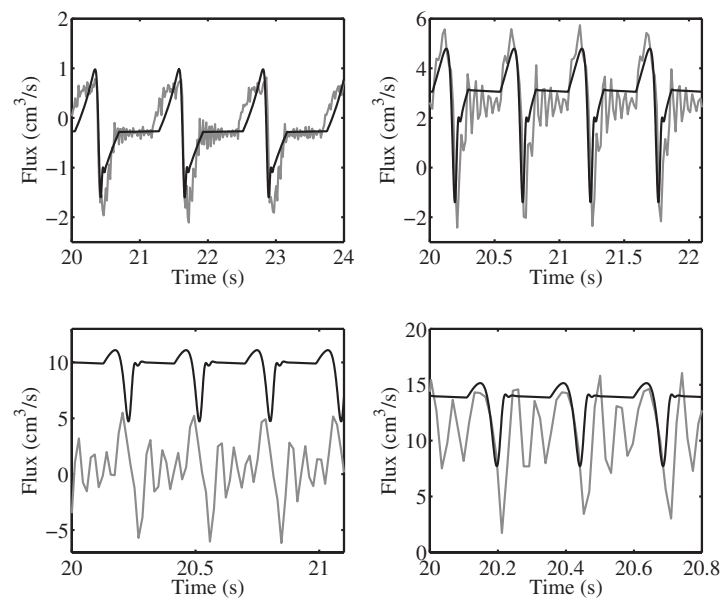

FIG. 9. Plots of $Q_{r}$ vs time. The black lines show model results. The gray lines show the experimental results. Upper left: $h_{0}=0.081 \mathrm{~cm}, L_{f}=5.66 \mathrm{~cm}$, and $f=0.81 \mathrm{~Hz}$. Upper right: $h_{0}=0.051 \mathrm{~cm}, L_{f}=5.66 \mathrm{~cm}$, and $f=1.91 \mathrm{~Hz}$. Lower left: $h_{0}=0.051 \mathrm{~cm}, L_{f}=3.27 \mathrm{~cm}$, and $f=3.49 \mathrm{~Hz}$. Lower right: $h_{0}$ $=0.051 \mathrm{~cm}, L_{f}=3.27 \mathrm{~cm}$, and $f=4.09 \mathrm{~Hz}$.

erates rapidly, then accelerates as the pumping region reopens. $Q_{r}$ is roughly constant when the forcing mechanism is not in contact with the pumping region, decreasing slightly in magnitude because of viscous drag. While the two fluxes are in phase during the deceleration as the tube is closed, the experiment's periods of acceleration and damped oscillation slightly precede the corresponding periods of acceleration and constant velocity of the model. At low frequencies, the motion of the forcing mechanism fails to be sinusoidal because of the strain caused by compressing the elastic tube. As a result, the downstroke takes longer and the upstroke shorter, which accounts for the phase discrepancies. The most glaring difference between the model and experimental results is the lack of damped oscillations in the model. Our approximation of the behavior of the elastic regions results in these oscillations being overdamped in the model, as described above in Sec. IV.

The upper right plot shows results obtained using the less rigid elastic tube, $f=1.91 \mathrm{~Hz}$, and $L_{f}=5.66 \mathrm{~cm}$. Again, the model and experiment are in good qualitative and quantitative agreement. At this larger frequency, there are only small differences in the phase over the entire pumping period. Again, the model does not capture the damped oscillations in $Q_{r}$. The lower left plot is for the less rigid elastic tube, $f=3.49 \mathrm{~Hz}$, and $L_{f}=3.27 \mathrm{~cm}$. The experiment shows a resonance at this frequency (discussed in Sec. III) that is characterized by a decreased mean flow and a phase locking of the forcing and damped oscillation. This resonance is not captured by the model, which predicts fluxes much greater than those observed in the experiment. The lower right plot shows results when we increase the frequency slightly to $4.09 \mathrm{~Hz}$ leaving other parameters unchanged. The model and experimental results are once again in reasonably good agreement. The damped oscillation in the experiment is now large compared to the features predicted by the model, but the latter can still be identified. At even larger frequencies,
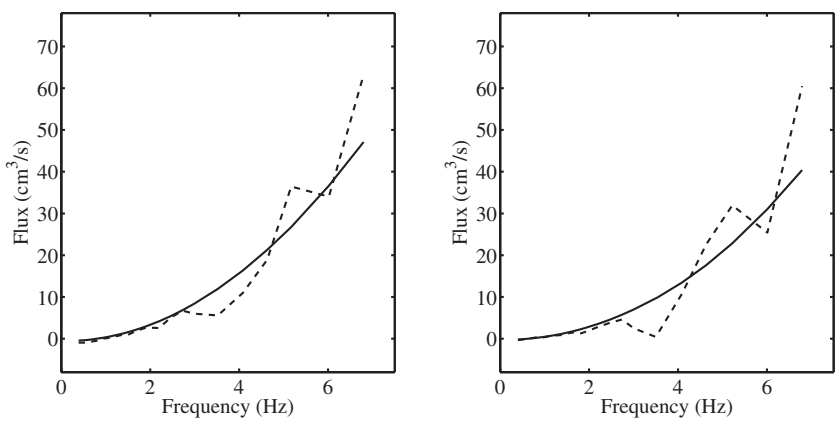

FIG. 10. Plots of $\bar{Q}$ vs forcing frequency for the less rigid elastic tube. The solid lines show the model results. The dashed lines show the experimental results. Left: $L_{f}=5.66 \mathrm{~cm}$. Right: $L_{f}=3.27 \mathrm{~cm}$.

which we do not picture, the damped oscillation becomes the dominant feature in the experimental data.

We now investigate whether the average flux obtained by the model agrees with the experiment as we vary the elastic tube rigidity, the forcing offset, and the forcing frequency. Figure 10 shows $\bar{Q}$ as a function of frequency for the less rigid elastic tube. In the plot on the left, $L_{f}=5$.66. In the plot on the right, $L_{f}=3.27$. The solid lines show the model results, and the dashed lines show the experimental results. In both plots, the model is in good qualitative and quantitative agreement with the experiment. In both, increasing the frequency increases the average flux, and both curves are convex. At small frequencies, both model and experiment have negative average fluxes. The model does not capture the dip at the resonance frequency of $3.5 \mathrm{~Hz}$. It also does not capture the irregularity observed at large frequencies that may be the result of resonances.

Figure 11 shows the average flux as a function of forcing offset for the more rigid elastic tube. In the plot in the upper left, $f=0.81 \mathrm{~Hz}$; in the upper right, $f=1.38 \mathrm{~Hz}$; in the lower
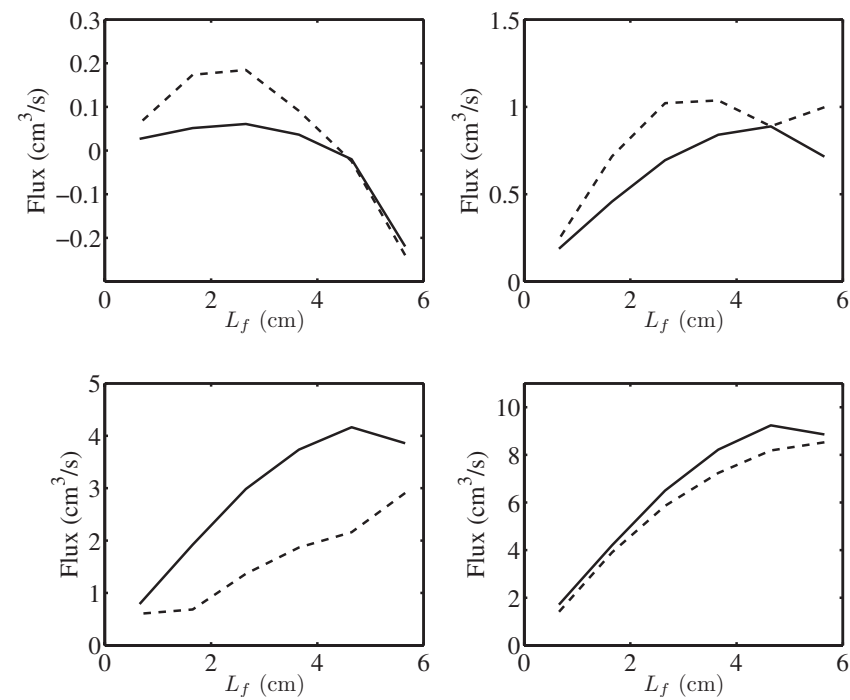

FIG. 11. Plots of $\bar{Q}$ vs forcing offset for the more rigid elastic tube. The solid lines show the model results. The dashed lines show the experimental results. Upper left: $f=0.81 \mathrm{~Hz}$. Upper right: $f=1.38 \mathrm{~Hz}$. Lower left: $f$ $=2.48 \mathrm{~Hz}$ (the resonance frequency). Lower right: $f=3.55 \mathrm{~Hz}$. 
left, $f=2.48 \mathrm{~Hz}$; and in the lower right, $f=3.55 \mathrm{~Hz}$. For all frequencies, the model captures the qualitative behavior of the average flux as a function of $L_{f}$. At $0.81 \mathrm{~Hz}$, the average flux in the model, like the experiment, is positive for small $L_{f}$ and negative for larger $L_{f}$. At $1.38 \mathrm{~Hz}$, the model's average flux increases, levels off, and then decreases as $L_{f}$ increases. At the resonance frequency of $2.48 \mathrm{~Hz}$, the model's average flux is well above that of the experiment, indicating that the model does not capture the resonance effects. Finally, at $3.55 \mathrm{~Hz}$, the agreement is again very good.

\section{ANALYSIS OF THE MODEL}

Our model produces results that, under a broad range of conditions, agree well with the experimental data. We now attempt to analyze the model so as to identify the physical mechanisms that generate the pumping and to produce a closed-form expression for the average flux. We make use of the fact that some nondimensional parameters in the model are small in the regime tested in the experiment.

We use nondimensional variables to identify the small parameters. The independent variables in the model equations are fluxes and volumes, so to nondimensionalize, we need to identify a volume scale and a time scale. We scale time so that the pumping period is 1 . We scale volume so that the volume of fluid ejected from the pumping region is 1 . Let $\Delta V=\max \left(V_{p, 0}-V_{p}\right)$. Then, the dimensionless variables are defined as

$$
\begin{aligned}
& t=\frac{1}{f} \tilde{t}, \quad V_{d}=\Delta V \widetilde{V}_{d}, \\
& Q_{r}=f \Delta V \widetilde{Q}_{r}, \quad Q_{p}=f \Delta V \widetilde{Q}_{p} .
\end{aligned}
$$

We also nondimensionalize $V_{p}$ by defining $\phi$ to be $V_{p} / V_{p, 0}$, so that $1-\Delta V / V_{p, 0} \leqslant \phi(t) \leqslant 1$.

Rewriting the equations of the model in dimensionless variables and dropping the tildes produces

$$
\begin{aligned}
\dot{V}_{d}= & Q_{r}-Q_{p}, \\
\dot{Q}_{r}= & -\zeta\left[V_{d}+\chi \dot{V}_{d}-\lambda\left(1-\phi-\chi \phi_{t}\right)\right]-\eta_{r} Q_{r}, \\
\dot{Q}_{p}= & 2 \gamma\left(\phi_{t} / \phi\right) Q_{p}+(\zeta \phi / \epsilon \alpha)\left[V_{d}+\chi \dot{V}_{d}-\lambda\left(1-\phi-\chi \phi_{t}\right)\right] \\
& -(\beta / \phi) \eta_{p} Q_{p} .
\end{aligned}
$$

The dimensionless constants are defined by

$$
\begin{aligned}
& \zeta=\frac{A_{r} k}{\rho L_{r} f^{2}}\left(\frac{1}{L_{1}}+\frac{1}{L_{2}}\right), \quad \lambda=\frac{V_{p, 0}}{\Delta V} \frac{L_{f}}{L_{1}+L_{2}}, \\
& \chi=\frac{n f}{k}, \quad \epsilon=\frac{A_{r} L_{p}^{2}}{V_{p, 0} L_{r}}, \\
& \eta_{r}=\frac{8 \pi \nu}{A_{r} f}, \quad \eta_{p}=\frac{8 \pi \nu L_{p}}{V_{p, 0} f} .
\end{aligned}
$$

See Table II for representative values of these constants for our experiments.
TABLE II. Values of dimensionless constants for $h_{0}=0.051 \mathrm{~cm}, L_{f}$ $=5.66 \mathrm{~cm}$, and $f=2.5 \mathrm{~Hz}$.

\begin{tabular}{lc}
\hline \hline Constant & Value \\
\hline$\zeta$ & 202.0 \\
$\lambda$ & 0.412 \\
$\chi$ & 0.0159 \\
$\epsilon$ & 0.0179 \\
$\eta_{r}$ & 0.0353 \\
$\eta_{p}$ & 0.0353 \\
\hline
\end{tabular}

The constant $\zeta$ is the dimensionless rigidity of the elastic regions. The constant $\lambda$ is the dimensionless asymmetry of the forcing divided by the fraction of the pumping region's volume that is ejected. The constant $\chi$ is the ratio of the response time of the elastic regions to the pumping period. For given pressures in the elastic regions, $\epsilon$ is the ratio of the acceleration of the fluid in the rigid tube to acceleration of the fluid in the pumping region. When, as in our case, the rest cross-sectional areas of the two regions are the same, $\epsilon$ is the length of the pumping region divided by the length of the rigid tube. Finally, $\eta_{r}$ and $\eta_{p}$ are ratios of the pumping period to the viscous decay times in the rigid tube and pumping region, respectively.

To perform asymptotics, we make use of the fact that $\epsilon$ and $\eta_{r}=\eta_{p}$ are small parameters. That $\chi$ is small is of no consequence. In the limit as $\epsilon$ approaches zero with other constants fixed, pressure differences in the elastic region accelerate fluid in the pumping region much faster than in the rigid tube, so the fluid in the rigid tube remains static and there is no pumping. In the limit as $\eta_{r}=\eta_{p}$ approach zero with other constants fixed, no viscous force damps the flow, which will accelerate to larger and larger speeds. We identify the distinguished limit of $\epsilon, \eta_{r}$, and $\eta_{p}$ approaching zero at a fixed ratio. The small deceleration due to viscosity balances the small acceleration in the rigid tube, and the solution converges to a finite answer in the limit.

We thus scale $\eta_{r}=\epsilon \eta_{r}^{0}$ and $\eta_{p}=\epsilon \eta_{p}^{0}$. We expand our solution as a power series in $\epsilon$ of the form $V_{d}=V_{d}^{0}+\epsilon V_{d}^{1}+\cdots$ and likewise for $Q_{r}$ and $Q_{p}$. We insert these expansions into the nondimensionalized equations [Eq. (14)] and equate powers of $\epsilon$. We know that the model, from rest, approaches a periodic steady state, so we look for a solution that is periodic in time. We assume for now that $\alpha, \beta$, and $\gamma$ are of order 1 . We later comment on what happens when these quantities become large, as they do when using the expression for $A_{p}$ specified in Eq. (9). We also assume that $V_{p}$ does not become very small so that $\phi$ is bounded away from zero.

At negative first order,

$$
V_{d}^{0}+\chi \dot{V}_{d}^{0}-\lambda\left(1-\phi-\chi \phi_{t}\right)=0,
$$

which allows us to solve for $V_{d}^{0}$,

$$
V_{d}^{0}=\lambda(1-\phi)+C_{0} e^{-t / \chi} .
$$

Periodicity requires $C_{0}=0$. At leading order, the fluid distributes itself so that the pressure in regions 1 and 2 is equal.

At zeroth order, the expression for $\dot{Q}_{r}^{0}$ becomes 


$$
\dot{Q}_{r}^{0}=0
$$

So, $Q_{r}^{0}$ is a constant that we call $\bar{Q}^{0}$. This constant will be determined by insisting that $Q_{r}^{1}$ be periodic. $\bar{Q}^{0}$ is the approximation of the average flux in the rigid tube that we seek. The expression for $\dot{V}_{d}^{0}$ gives

$$
Q_{p}^{0}=Q_{r}^{0}-\dot{V}_{d}^{0}=\bar{Q}^{0}+\lambda \phi_{t} .
$$

Now, we find $V_{d}^{1}$,

$$
\frac{\zeta \phi}{\alpha}\left(V_{d}^{1}+\chi \dot{V}_{d}^{1}\right)=\dot{Q}_{p}^{0}-2 \gamma \frac{\phi_{t}}{\phi} Q_{p}^{0} .
$$

Finally, we arrive at an expression for $\dot{Q}_{r}^{1}$,

$$
\begin{aligned}
\dot{Q}_{r}^{1} & =-\zeta\left(V_{d}^{1}+\chi \dot{V}_{d}^{1}\right)-\eta_{r}^{0} \bar{Q}^{0} \\
& =-\frac{\alpha}{\phi}\left(\dot{Q}_{p}^{0}-2 \gamma \frac{\phi_{t}}{\phi} Q_{p}^{0}\right)-\eta_{r}^{0} \bar{Q}^{0} \\
& =\frac{\alpha}{\phi}\left(-\lambda \phi_{t t}+2 \gamma \frac{\phi_{t}}{\phi}\left(\bar{Q}^{0}+\lambda \phi_{t}\right)\right)-\eta_{r}^{0} \bar{Q}^{0} .
\end{aligned}
$$

We integrate in time over a pumping period and use the periodicity of $Q_{r}^{1}$. We assume that the pumping motion is symmetric in time, as it is approximately in our experiments, so that the $\alpha \gamma \phi_{t} / \phi^{2}$ term integrates to zero. Integrating the term involving $\phi_{t t}$ once by parts,

$$
\bar{Q}^{0}=\frac{\lambda}{\eta_{r}^{0}} \int_{0}^{1}(2 \gamma-1) \alpha \frac{\phi_{t}^{2}}{\phi^{2}}+\frac{\alpha_{t} \phi_{t}}{\phi} d t .
$$

Redimensionalizing $\bar{Q}^{0}$, we have an approximation to the average flux in the rigid tube,

$$
\bar{Q}^{0}=\frac{f^{2} A_{r}^{2} L_{f} L_{p}^{2}}{8 \pi \nu\left(L_{1}+L_{2}\right) L_{r}} C_{g},
$$

where $C_{g}$ is a nondimensional constant which depends only on the shape of the pumping region over the forcing period,

$$
C_{g}=\frac{1}{f} \int_{0}^{1 / f}(2 \gamma-1) \alpha \frac{\dot{V}_{p}^{2}}{V_{p}^{2}}+\alpha_{t} \frac{\dot{V}_{p}}{V_{p}} d t
$$

From Eq. (18), we see how the average flux in the pump depends on various parameters. For fixed forcing shape, the average flux is always in the same direction, increasing as the square of the frequency, and increasing linearly as the forcing position moves away from the center of the elastic tube. The average flux is quadratic in the cross-sectional area of the rigid tube and in the length of the pumping region. It is inversely proportional to the viscosity of the fluid and to the length of the rigid tube.

There is no dependence at all on either $E$ or $Y$, meaning that there is no dependence on the elastic properties of the rigid tube. We expect this result to be valid only for elastic tubes for which our assumptions in deriving the model are valid. Very soft elastic tubes may have wave speeds small enough that wave phenomena ignored by this model become important. Also, while $E$ and $Y$ may not affect the steady-
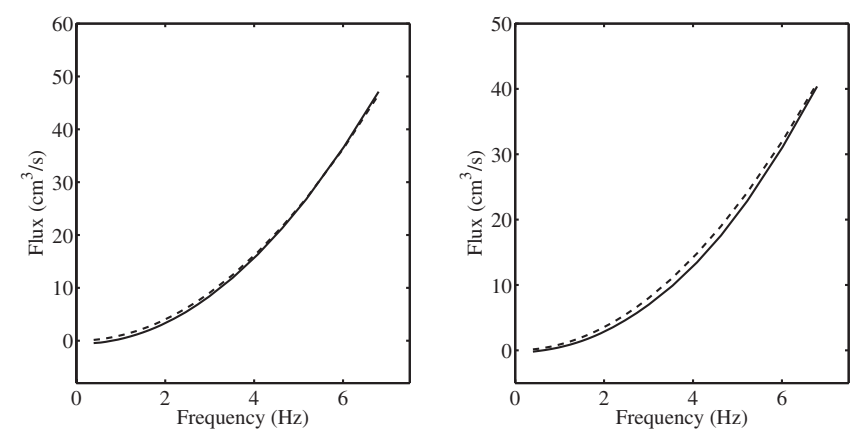

FIG. 12. Comparison of the average flux generated by the full model (shown by the solid lines) with that predicted by the asymptotic formula in Eq. (18) (shown by the dashed lines). Both plots are for the less rigid elastic tube. Left: $L_{f}=5.66 \mathrm{~cm}$, and we have chosen $C_{g}=2.1$ in the asymptotic formula to fit the results of the model. Right: $L_{f}=3.22 \mathrm{~cm}$, and we have chosen $C_{g}=3.2$ to fit the results of the model.

state average flux, they will affect the time to reach steady state, the fluctuations in time about the average flux, and the mechanical efficiency of the pump.

The dependence of the average flux on the shape of the forcing, including the dependence on $\Delta V$, is encapsulated in $C_{g}$, which depends on the forcing shape in a complicated way. When the pumping region is a cylinder at all times with a sinusoidally varying radius, we can calculate $C_{g}$ explicitly. In this case, $\alpha, \beta$, and $\gamma$ are identically 1 . Then,

$$
C_{g}=4 \pi^{2}\left(\frac{1-\Delta V / 2 V_{p, 0}}{\sqrt{1-\Delta V / V_{p, 0}}}-1\right) .
$$

For small $\Delta V$ compared to $V_{p, 0}, C_{g} \approx \pi^{2} \Delta V^{2} / 2 V_{p, 0}^{2}$, so the average flux increases as the square of $\Delta V$. $C_{g}$ diverges as $\Delta V$ increases to $V_{p, 0}$. However, in this case, our asymptotics break down because $\phi$ is not bounded away from zero. Using this expression for $C_{g}$, Eq. (18) agrees with $\bar{Q}$ obtained by integrating the full model (with a cylindrical pumping region) with approximately $10 \%$ error over a wide range of parameters.

When we use $A_{p}$ specified in Eq. (9), $\alpha, \beta$, and $\gamma$ diverge when the tube comes to a full close. When these factors are large, the asymptotic analysis above is no longer valid. In a very small interval of time, close to when the tube comes to a full close, the viscous term in Eq. (7) dominates because $\beta$ is very large, even compared to $\gamma / \epsilon$. We have not yet fully developed an asymptotics that deals with this case. The scaling of $\bar{Q}^{0}$ should be approximately the same with the other parameters, but we cannot explicitly calculate $C_{g}$. Instead, $C_{g}$ should be thought of as some positive constant of order 1.

This claim is verified in Fig. 12, which shows the average fluxes obtained with the full model (solid lines) and those of the asymptotic formula given in Eq. (18) (dashed lines). The plot on the left is for $L_{f}=5.66 \mathrm{~cm}$. To generate the flux given by the asymptotic formula in this plot, we have chosen $C_{g}=2.1$, which results in a very good fit. The plot on the right is for $L_{f}=3.27 \mathrm{~cm}$. For this plot, we have chosen $C_{g}=3.2$. While the flux has approximately the predicted behavior as a function of frequency, we need to adjust $C_{g}$ slightly when we change $L_{f}$ because of the breakdown in our 


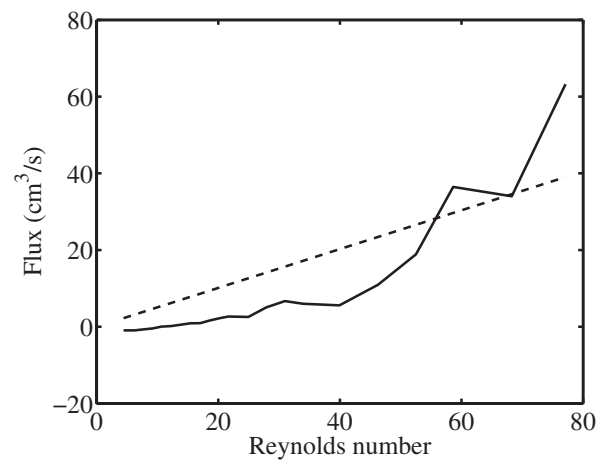

FIG. 13. The solid line shows $\bar{Q}$ vs Reynolds number for the less rigid elastic tube and $L_{f}=5.66 \mathrm{~cm}$. The dashed line shows the flux of a traditional pump operating at the same frequency which displaces a volume $A_{r} L_{p}$ in every period.

asymptotics when the tube comes to a full close. Note that our asymptotic formula always predicts an average flux in the positive direction, while the full model captures the direction reversals at low frequencies.

The expression for the average flux is perhaps more intuitively understood if the terms are grouped in the form

$$
\bar{Q}^{0}=\left(A_{r} L_{p} f\right)\left(\frac{A_{r} f}{8 \pi \nu}\right)\left(\frac{L_{p}}{L_{r}}\right)\left(\frac{L_{f}}{L_{1}+L_{2}}\right) C_{g} .
$$

The first term in parentheses is a flux, namely, the flux required to eject all the fluid from the pumping region in one pumping period. The second term in parentheses is a frequency Reynolds number, the third term is the fraction of the tube that is squeezed in pumping, and the fourth term is the dimensionless asymmetry of the pumping.

Using this expression, we can now comment on the efficiency of valveless pumping. During each period of the forcing, a volume on the order of $A_{r} L_{p}$ must be ejected from the pumping region. A traditional pump that ejected this volume in only the desired direction over the same period would have an average flux of $A_{r} L_{p} f$, the first term in Eq. (20). A measure of efficiency of valveless pumping relative to traditional pumping is the ratio of the flux produced by the former to that produced by the latter. This ratio is given by the final four terms in Eq. (20). Assuming that $C_{g}$ and the forcing asymmetry are of order 1 , one needs to be at a Reynolds number larger than 1 over the fraction of the tube squeezed for valveless pumping to be efficient. In the case of our experiment, this estimate implies a Reynolds number of order of $50-100$.

Figure 13 validates this estimate. The figure shows the experimentally measured flux as a function of Reynolds number (solid line) for $L_{f}=5.66 \mathrm{~cm}$ and the less rigid elastic tube against the flux of a traditional pump of the same frequency that displaces a volume $A_{r} L_{p}$ in every period (dashed line). At low Reynolds numbers, the flux of a traditional pump is well above that of a valveless pump, but at Reynolds numbers greater than about 60, the valveless pump surpasses the traditional pump. It seems that it is possible for a valveless pump to be quite efficient but only at high Reynolds numbers. Valveless pumping does not seem to be an efficient mechanism for fetal blood circulation or for micropumps. We emphasize that the efficiency we consider is the total flux per volume displaced, not traditional mechanical efficiency.

\section{PHYSICAL EXPLANATION}

The physical mechanism that creates valveless pumping has long been a mystery. Why is it that asymmetric forcing creates a net flow, and why is it that the flow goes in the observed direction? In this section, we attempt to answer these questions using our model. This section will show that there is a quantity that we call $J$ which obeys a simple evolution equation and which is approximately proportional to the flux in the rigid tube. Recall that the momentum equation for flow in a long thin tube [Eq. (3)] is a conservation law for velocity. We let $J$ be the integral of the fluid velocity over the rigid section and pumping region. We will derive an evolution equation for $J$ showing that it can increase or decrease only in response to nonlinear advective fluxes of velocity into and out of the pumping region. The relative magnitudes of these fluxes determine the sign of $J$, and we then show that $J$, and thus $Q_{r}$, will be driven in the direction observed by Liebau.

Using Eq. (5) for the velocity in the pumping region, we find that the integral of the fluid velocity $J=L_{r} Q_{r} / A_{r}$ $+L_{p} Q_{p}\left\langle A_{p}^{-1}\right\rangle$. We first claim that $Q_{r}$ is nearly proportional to $J$ with a positive constant of proportionality. The reason is that $L_{p}$ is much less than $L_{r}$, so $Q_{r}$ is $A_{r} J / L_{r}$ plus a small correction. Also, the time average of $Q_{r}$ must equal that of $Q_{p}$ for a periodic solution, so the time averages of $J$ and $Q_{r}$ must, to an even greater degree, be approximately proportional. Therefore, if we know that $J$ grows in a particular direction, $Q_{r}$ must grow in the same direction.

Differentiating the equation for $J$ and using Eqs. (4) and (7), we find a simple equation for the evolution of the integral of the velocity,

$$
\dot{J}=\frac{Q_{1}^{2}-Q_{2}^{2}}{2 A_{p}\left(L_{p} / 2, t\right)^{2}}-8 \pi \nu\left(\frac{L_{r}}{A_{r}^{2}} Q_{r}+L_{p}\left\langle A_{p}^{-2}\right\rangle Q_{p}\right) .
$$

Ignoring viscous drag, velocity is conserved in the pumping region and rigid section, so $J$ can only change because of velocity fluxes at the endpoints. The pressure part of the velocity flux into region 1 from the rigid section and that out of region 1 to the pumping region are equal and opposite, so they have no net effect on $J$. The same is true for region 2, and all the pressure terms cancel. A pressure difference in regions 1 and 2 does not affect the integral of the velocity.

The velocity into and out of the rigid section must be equal, so the advective flux of velocity into the rigid section cancels the advective flux out. The same is not true of the pumping region, since its volume can change. The advective velocity flux into the pumping region is $\left[Q_{1} / A_{p}\left(L_{p} / 2, t\right)\right]^{2} / 2$. The flux out is $\left[Q_{2} / A_{p}\left(L_{p} / 2, t\right)\right]^{2} / 2$. The difference of these creates or destroys velocity, increasing or decreasing $J$. When the volume of the pumping region does not change, they cancel and $J$ is constant.

If the nonlinear term in the momentum equation [Eq. (3)] is omitted, there will be no advective velocity flux and $J$ will be conserved absent viscosity. Numerical experiments 
confirm that there is no net flow in this case. This reinforces our claim that this valveless pumping mechanism does not work at small Reynolds numbers, when the nonlinear term is negligible. We expect this result because symmetric movements in a Stokes flow cannot produce locomotion or, equivalently, pumping. ${ }^{19,20}$

We have shown that differences in the advective fluxes of velocity into and out of the pumping region control the growth of $J$, and thus $Q_{r}$. This is the physical reason for flow creation in the pump. We now show why $Q_{r}$ tends to grow in the direction observed by Liebau. If $Q_{1}$ tends to be of larger magnitude than $Q_{2}, J$ will become increasingly positive. If $Q_{2}$ tends to be of larger magnitude than $Q_{1}, J$ will become increasingly negative. The direction for the valveless pump is selected by the fact that the magnitude of the flux to or from the pumping region tends to be larger in the direction of the more compliant reservoir, here meaning the longer of regions 1 and 2. Because the pumping region tends to exchange fluid with the more compliant reservoir, the flow tends to be toward the less compliant reservoir.

In the case when $L_{f}>0$, region 1 is longer and thus more compliant. During closing, more fluid is ejected into region 1, making $Q_{1}$ more negative than $Q_{2}$ is positive. During opening, more fluid is sucked in from region 1, making $Q_{1}$ more positive than $Q_{2}$ is negative. Both of these effects increase $J$. Thus, $Q_{r}$ becomes positive on average, which is the direction of flow observed by Liebau and in our experiment.

The difference between $Q_{1}$ and $Q_{2}$ comes about because of the different pressures created in regions 1 and 2 . The pressure differences modify the flow through the pumping region. We saw in the asymptotics section that the details of the pressures are irrelevant, a result confirmed by the experimental observation that the thickness of the elastic tube makes little difference (apart from resonances). We now see why. The magnitudes of the pressure differences do not enter into Eq. (21). What does matter is that these pressures affect $Q_{1}$ and $Q_{2}$, so that the fluid distributes itself in a certain way, namely, so that the pressures in the two regions are approximately equalized.

Also, details of the rigid section are unimportant, except insofar as they affect the multiplier by which $Q_{r}$ can be found from $J$. We expect that the valveless pump can be connected to an arbitrary fluid circuit, even a thin capillary tube, with similar results. A flow will be created in the direction of the less compliant region, and the strength of the flow, in periodic steady state, will be inversely proportional to the viscous resistance of the fluid circuit. An important requirement for this analysis to be valid is that the pumping region always exchanges fluid preferentially with the more compliant reservoir. If not, the results may be more complicated but should be comprehensible by analyzing with which reservoir the pumping region does preferentially exchange fluid.

We have so far ignored the viscous term in Eq. (21). When $A_{p}$ is identically $A_{r}$, the viscous term is $-8 \pi \nu J / A_{r}$. More generally, viscosity tends to decrease the magnitude of $J$. A periodic steady state is achieved when this decay matches the forcing described above.

Exceptions may occur when $A_{p}$ departs significantly from $A_{r}$. We think this effect accounts for the reversals of
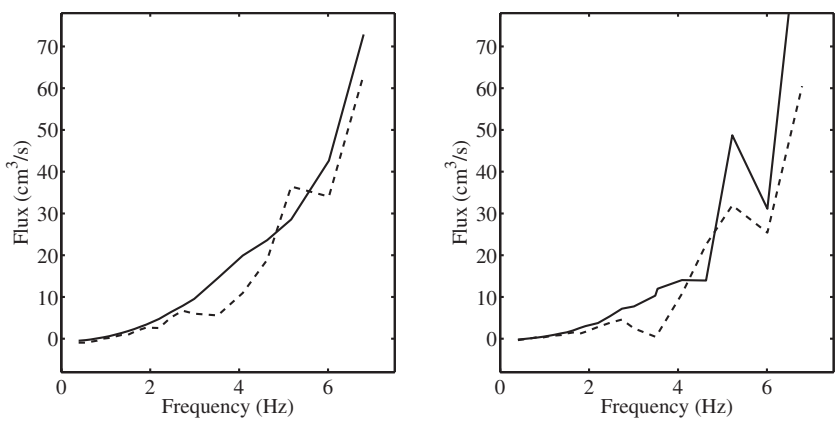

FIG. 14. Plots of $\bar{Q}$ vs forcing frequency for the less rigid elastic tube with $Y$ reduced from its experimentally measured value by a factor of 10 . The solid lines show the model results. The dashed lines show the experimental results. Left: $L_{f}=5.66 \mathrm{~cm}$. Right: $L_{f}=3.27 \mathrm{~cm}$. Particularly in this second plot, resonancelike effects can be seen in the model.

direction that we see in the experiment and the model at low frequencies, when the advective fluxes are weak. As the pumping region comes to a full close, the system briefly becomes a simple oscillator in which fluid moves between regions 1 and 2 via the rigid tube. If the length of time during which the pumping region is nearly closed is on the order of the period of this oscillation (whose frequency is $20-35 \mathrm{~Hz}$ ), direction reversals may occur. Since the pumping region is nearly closed for only a fraction of the pumping cycle, we see these direction reversals at pumping frequencies around $1 \mathrm{~Hz}$.

In summary, we have used the conservation properties of the tube equations to motivate the definition of a quantity, $J$, that obeys a simple evolution equation. The equation indicates that $J$ will increase in the opposite direction with which the pumping region prefers to exchange fluid during opening and closing. The pumping region prefers to exchange fluid with the more compliant reservoir, so $J$ will be toward the less compliant reservoir. Viscous drag creates a periodic steady state. Knowing $J$, we know that $Q_{r}$ is approximately proportional to and is in the same direction as $J$.

A final question is the source of the resonances we observe in the experiment. Evidence suggests that these are related to the damped oscillations in $Q_{r}$. First, the resonances seem to happen when the durations of the accelerations that occur when the pumping region is closed and opened match a half-period of the damped oscillations, as shown in Fig. 3. Second, the model captures all the features of the time series of $Q_{r}$ except for the damped oscillations, and the model results agree well with those from the experiment except at resonances. As a final test of this theory, we artificially create damped oscillations in the model by decreasing $Y$ by a factor of 10. Results can be seen in Fig. 14. Compare with Fig. 10. On one hand, we see that decreasing $Y$ does not affect much the average flux in the model. On the other hand, we see irregularities in the flux as a function of frequency that look very much like those caused by resonances in the experiment. This is further proof that resonances are the result of coupling of the damped oscillations with the behavior described in the model. As mentioned before, we feel that the failure of our model to exhibit damped oscillations is related to the fact that the time scale of the oscillation of fluid be- 
tween regions 1 and 2 via the pumping region is on the order of the wave time scale. It is not clear if this problem could be corrected while maintaining the model's simplicity and accuracy. A one-dimensional or higher model that fully incorporates wave dynamics in the elastic tube may be necessary.

\section{CONCLUSION}

We have constructed a valveless pump and have carefully measured the time series of velocities inside the loop for various values of important parameters. We have confirmed the observation of Liebau that asymmetric forcing creates a net flow around the pump. Except at low frequencies, the flow is in the direction observed by Liebau. Generally, higher frequencies result in faster average velocities, but we also observed resonances and flow reversals. We did not observe flow reversals at high frequencies, which have been reported by other authors in somewhat similar experiments ${ }^{9}$ and in numerical simulations. ${ }^{5}$ In our experiment, flow in the direction observed by Liebau is robust.

We have also constructed a model that reproduces many experimental results, both for the average flux in the pump and also for the time series of the flux in the rigid part of the pump. The model is derived constructively so that we know the validity of the approximations involved, and all model parameters are measured experimentally. The model does not reproduce the damped oscillations seen the experiment, which seem to be related to resonances. The model is valid in the regime where the pumping period is much longer than the time for a wave to travel the length of the elastic tube, which is the regime tested by our experiment. The model may break down if the forcing frequency is much greater or if the elastic tube is much longer or much more flexible.

Our simple model can be solved using an asymptotic approximation in a particular distinguished limit. This limit corresponds to large Reynolds number and a large ratio of the length of the rigid tube to the length of the pumping region. An approximate expression for the average flux has been derived, which reveals the dependence of the average flux on the parameters of the experiment. We have found that our valveless pump can be quite efficient at high Reynolds number but is inefficient at low Reynolds number. Further study is needed to determine if our results apply to the valveless micropump experiment of Rinderknecht $e t a l^{7}$ or if a different pumping mechanism operates.

In our analysis, we have found that the key quantity to consider is the integral of the velocity in the pump. Velocity is approximately conserved throughout the tube ring, and the integral of the velocity is not affected by pressure differences that may develop. This integral may increase or decrease because of nonlinear advection of velocity to or from the regions to the left and right of the pumping region, where the elastic tube is compressed. Pressure differences cause the pumping region to exchange fluid preferentially with the longer uncompressed portion of the elastic tube. This increases the integral of the velocity in the direction of the shorter portion, which must result in an average flow toward the shorter portion, the observed direction.
One way to conceive of this result is that momentum is lost as the fluid ejected from the pumping region into the longer uncompressed region collides inelastically with the fluid therein. Momentum is lost in the direction of the longer uncompressed region, so the leftover momentum drives the flow toward the shorter region. Of course, we have shown that it is velocity, not momentum, that must be considered.

The details of the pressure differences created, as well as the details of the rigid section, are irrelevant. We conjecture that a similar valveless mechanism could be used as a pump in an arbitrary fluid circuit and that the induced flow would be toward the shorter portion of the elastic tube and have a magnitude proportional to the viscous resistance of the fluid circuit. (The direction could change if the squeezed region does not preferentially exchange fluid with the longer portion of the elastic tube, which may happen if the circuit is not volume conserving.)

Our model shows that we can explain many of the features of valveless pumping in the regime tested in our experiment without modeling the detailed behavior of waves in the elastic tube. This implies that valveless pumping is not itself an effect of resonance. Resonances that modify the behavior of the flow do occur, and wave phenomena certainly play a role in generating these resonances. The detailed wave behavior will be important in the regime where our model breaks down.

Improvements to the model could include a more realistic characterization of the viscous drag in the rigid tube, a better characterization of the pumping region and the boundary conditions at the junction of the pumping region and regions 1 and 2, and an addition of the effect of waves in the elastic tube that create damped oscillations. We would like, in future work, to improve the asymptotics to account for what happens when the pumping region closes completely, as it does in the experiment. We would also like to investigate the mechanical efficiency of the pump using our model. Experimentally, we would like to test valveless pumps in different regimes to see if our model remains valid and, if not, what additional mechanisms are at work. We would also like to examine the resonances more thoroughly.

Nevertheless, we have identified an essential physical mechanism responsible for valveless pumping. An understanding of this and other mechanisms can be used to determine the role of valveless pumping in biological and engineering applications. The scaling relationship predicted by our model between the average pumping flux and various other parameters should have wide applicability. Our model can be used to predict the exact fluxes of similar pumps with different parameters, and can be used to designs pumps with desired properties. Similar models may be successful in modeling pumps with slightly different characteristics, for instance, that used in the experiment by Hickerson et al. in which flow reversals are seen at high frequencies. ${ }^{9}$

\section{ACKNOWLEDGMENTS}

We would like to thank Charles Peskin and Michael Shelley for helpful insights and suggestions. This research is supported by Department of Energy Grant No. DE- 
FG0288ER25053 and National Science Foundation Grant No. DMS-9980069.

\section{APPENDIX: DERIVATION OF THE EQUATION FOR $Q_{p}$}

We integrate the momentum equation (3) from $-L_{p} / 2$ to $L_{p} / 2$, the extent of the pumping region,

$$
\begin{aligned}
& \int_{-L_{p} / 2}^{L_{p} / 2} u_{t} d x+\frac{1}{2}\left[u\left(\frac{L_{p}}{2}, t\right)^{2}-u\left(\frac{-L_{p}}{2}, t\right)^{2}\right]+\frac{p_{2}-p_{1}}{\rho} \\
& =-8 \pi \nu \int_{-L_{p} / 2}^{L_{p} / 2} \frac{u}{A_{p}} d x .
\end{aligned}
$$

Recall that the velocity $u$ in the pumping region is given by Eq. (5),

$$
u=\frac{Q_{p}(t)}{A_{p}}-\frac{1}{A_{p}} \int_{0}^{x} \partial_{t} A_{p}\left(x^{\prime}, t\right) d x^{\prime}
$$

and that $A_{p}$ is assumed to be an even function of $x$ for all $t$. Thus, the second term in this equation is odd for all $t$. We insert this expression for $u$ into the integrated momentum equation to derive an equation for $Q_{p}$.

We first introduce a bracket notation to represent averaging over the pumping region. For a function $f(x)$, we define

$$
\langle f\rangle=\frac{1}{L_{p}} \int_{-L_{p} / 2}^{L_{p} / 2} f(x) d x
$$

Using the evenness of $A_{p}$, we find simplified expressions for the terms in the integrated momentum equation,

$$
\begin{aligned}
& \int_{-L_{p} / 2}^{L_{p} / 2} u_{t} d x=L_{p}\left(Q_{p}\left\langle A_{p}^{-1}\right\rangle+Q_{p}\left\langle\partial_{t} A_{p}^{-1}\right\rangle\right), \\
& u\left(\frac{L_{p}}{2}, t\right)^{2}-u\left(\frac{-L_{p}}{2}, t\right)^{2}=-2 L_{p} Q_{p} A_{p}\left(L_{p} / 2\right)^{-2}\left\langle\partial_{t} A_{p}\right\rangle, \\
& \int_{-L_{p} / 2}^{L_{p} / 2} \frac{u}{A_{p}} d x=L_{p} Q_{p}\left\langle A_{p}^{-2}\right\rangle .
\end{aligned}
$$

Inserting these expressions into the momentum equation and dividing by $L_{p}\left\langle A_{p}^{-1}\right\rangle$, so that we get an equation for $\dot{Q}_{p}$, we find

$$
\begin{aligned}
\dot{Q}_{p}= & \left(-\frac{\left\langle\partial_{t} A_{p}^{-1}\right\rangle}{\left\langle A_{p}^{-1}\right\rangle}+\frac{\left\langle\partial_{t} A_{p}\right\rangle}{A_{p}\left(L_{p} / 2\right)^{2}\left\langle A_{p}^{-1}\right\rangle}\right) Q_{p}+\frac{p_{1}-p_{2}}{\rho L_{p}\left\langle A_{p}^{-1}\right\rangle} \\
& -\frac{8 \pi \nu\left\langle A_{p}^{-2}\right\rangle}{\left\langle A_{p}^{-1}\right\rangle} Q_{p} .
\end{aligned}
$$

We identify dimensionless functions of time that characterize the shape of the pumping region and that allow us to express the coefficients in Eq. (A1) in a simple way that separates the effects of the pumping region shape from those of its volume $V_{p}$ and the derivative of its volume $\dot{V}_{p}$,

$$
\begin{aligned}
& \alpha(t)=\left\langle A_{p}\right\rangle\left\langle A_{p}^{-1}\right\rangle, \\
& \beta(t)=\alpha\left\langle A_{p}^{-2}\right\rangle\left\langle A_{p}^{-1}\right\rangle^{-2}, \\
& \gamma(t)=\frac{\left\langle A_{p}\right\rangle}{2\left\langle A_{p}^{-1}\right\rangle\left\langle\partial_{t} A_{p}\right\rangle}\left(-\left\langle\partial_{t} A_{p}^{-1}\right\rangle+\frac{\left\langle\partial_{t} A_{p}\right\rangle}{A_{p}\left(L_{p} / 2, t\right)^{2}}\right) .
\end{aligned}
$$

These functions are discussed further in the text. Finally, we arrive at the expression for $\dot{Q}_{p}$ in Eq. (7),

$$
\dot{Q}_{p}=2 \gamma(t) \frac{\dot{V}_{p}}{V_{p}} Q_{p}+\frac{V_{p}}{\alpha(t) \rho L_{p}^{2}}\left(p_{1}-p_{2}\right)-\beta(t) \frac{8 \pi \nu L_{p}}{V_{p}} Q_{p}
$$

We have used that $\left\langle A_{p}\right\rangle=V_{p} / L_{p}$ and that $\left\langle\partial_{t} A_{p}\right\rangle=\dot{V}_{p} / L_{p}$.

${ }^{1}$ G. Liebau, "Über ein ventilloses pumpprinzip," Naturwiss. 41, 327 (1954).

${ }^{2}$ G. Liebau, "Die stromungsprinzipien des herzens," Z. Kreislaufforsch. 44, 677 (1955)

${ }^{3}$ G. Liebau, "Die bedeutung der tragheitskrafte für dynamik des blutkreislaufs," Z. Kreislaufforsch. 46, 428 (1957).

${ }^{4}$ T. J. Pedley, The Fluid Mechanics of Large Blood Vessels (Cambridge University Press, New York, 1980).

${ }^{5}$ E. Jung and C. S. Peskin, "Two-dimensional simulations of valveless pumping using the immersed boundary method," SIAM J. Sci. Comput. (USA) 23, 19 (2001).

${ }^{6}$ A. S. Forouhar, M. Liebling, A. I. Hickerson, A. Nasiraei-Moghaddam, H. J. Tsai, J. R. Hove, S. E. Fraser, M. E. Dickinson, and M. Gharib, "The embryonic vertebrate heart tube is a dynamic suction pump," Science 312, 751 (2006)

${ }^{7}$ D. Rinderknecht, A. I. Hickerson, and M. Gharib, "A valveless micro impedance pump driven by electromagnetic actuation," J. Micromech. Microeng. 15, 861 (2005).

${ }^{8} \mathrm{P}$. J. Kilner, "Formed flow, fluid oscillation and the heart as a morphodynamic pump," Eur. Surg. Res. 19, 89 (1987).

${ }^{9}$ A. I. Hickerson, D. Rinderknecht, and M. Gharib, "Experimental study of the behavior of a valveless impedance pump," Exp. Fluids 38, 534 (2005).

${ }^{10} \mathrm{H}$. Thomann, "A simple pumping mechanism in a valveless tube," $\mathrm{Z}$. Angew. Math. Phys. 29, 169 (1978).

${ }^{11}$ J. T. Ottesen, "Valveless pumping in a fluid-filled closed elastic tubesystem: one-dimensional theory with experimental validation," J. Math. Biol. 46, 309 (2003).

${ }^{12}$ C. G. Manopoulos, D. S. Mathioulakis, and S. G. Tsangaris, "Onedimensional model of valveless pumping in a closed loop and a numerical solution," Phys. Fluids 18, 017106 (2006).

${ }^{13}$ A. Borzì and G. Propst, "Numerical investigation of the Liebau phenomenon," Z. Angew. Math. Phys. 54, 1050 (2003).

${ }^{14}$ D. Auerbach, W. Moehring, and M. Moser, "An analytic approach to the Liebau problem of valveless pumping," Cardiovas. Eng. 4, 201 (2004).

${ }^{15}$ A. I. Hickerson and M. Gharib, "On the resonance of a pliant tube as a mechanism for valveless pumping," J. Fluid Mech. 555, 141 (2006).

${ }^{16}$ M. Moser, J. W. Huang, G. S. Schwarz, T. Kenner, and A. Noordergraaf, "Impedance defined flow: Generalisation of William Harvey's concept of the circuation-370 years later," Int. J. of Cardiovasc. Med. and Sci. 1, 205 (1998)

${ }^{17}$ We were unable to measure the complete velocity profiles for all of our experiments without introducing significant systematic errors, and therefore we were unable to directly measure the tube flux. Instead, we use a somewhat crude linear approximation: $Q(t)=C_{Q} A_{r} u(t)$. The constant $C_{Q}$ $=0.70$ is determined empirically by studying the complete time averaged velocity profiles for a subset of our experiments. These profiles are nearly 
parabolic but with regions of high shear at the tube's edges, indicating the presence of boundary layers. A plug flow would have $C_{Q}=1$, and a Poiseuille flow would have $C_{Q}=1 / 2$. Our approximation falls between these extremes. The error associated with this approximation is on the order of $5 \%-10 \%$, which is sufficiently small to compare our results to those of our simple model.
${ }^{18}$ J. Lighthill, Mathematical Biofluiddynamics (Society for Industrial and Applied Mathematics, Philadelphia, 1975).

${ }^{19}$ S. Childress and R. Dudley, "Transition from ciliary to flapping mode in a swimming mollusc: Flapping flight as a bifurcation in $R e_{\omega}$, "J. Fluid Mech. 498, 257 (2004).

${ }^{20}$ E. M. Purcell, "Life at low Reynolds number," Am. J. Phys. 45, 3 (1977). 